Publications of the Astronomical Society of the Pacific, 119: 102-111, 2007 January

(C) 2007. The Astronomical Society of the Pacific. All rights reserved. Printed in U.S.A.

\title{
The James Clerk Maxwell Telescope Spectral Legacy Survey
}

\author{
R. Plume, ${ }^{1}$ G. A. Fuller,${ }^{2}$ F. Helmich,${ }^{3,4}$ F. F. S. van der Tak ${ }^{3}$ H. Roberts,${ }^{5}$ J. Bowey, ${ }^{6}$ J. Buckle ${ }^{7}$ H. Butner ${ }^{8}$ \\ E. Caux,${ }^{9}$ C. Ceccarelli,${ }^{10}$ E. F. van Dishoeck,${ }^{11}$ P. Friberg,${ }^{8}$ A. G. Gibb,${ }^{12}$ J. Hatchell, ${ }^{13}$ M. R. Hogerheijde, ${ }^{11}$ \\ H. Matthews, ${ }^{14}$ T. J. Millar,${ }^{5}$ G. Mitchell, ${ }^{15}$ T. J. T. Moore, ${ }^{16}$ V. Ossenkopf,${ }^{3,4,17}$ J. M. C. Rawlings, ${ }^{6}$ J. Richer, ${ }^{7}$ \\ M. Roellig, ${ }^{17}$ P. Schilke, ${ }^{18}$ M. Spanns, ${ }^{4}$ A. G. G. M. Tielens, ${ }^{19}$ M. A. Thompson,${ }^{20}$ S. Viti, ${ }^{6}$ B. Weferling, ${ }^{8}$ \\ Glenn J. White, ${ }^{21,22}$ J. Wouterloot, ${ }^{8}$ J. YAtes, ${ }^{6}$ And M. Zhu ${ }^{8,14}$ \\ Received 2006 October 20; accepted 2006 November 27; published 2007 January 24
}

\begin{abstract}
Stars form in the densest, coldest, most quiescent regions of molecular clouds. Molecules provide the only probes that can reveal the dynamics, physics, chemistry, and evolution of these regions, but our understanding of the molecular inventory of sources and how this is related to their physical state and evolution is rudimentary and incomplete. The Spectral Legacy Survey (SLS) is one of seven surveys recently approved by the James Clerk Maxwell Telescope (JCMT) Board of Directors. Beginning in 2007, the SLS will produce a spectral imaging survey of the content and distribution of all the molecules detected in the $345 \mathrm{GHz}$ atmospheric window (between 332 and $373 \mathrm{GHz}$ ) toward a sample of five sources. Our intended targets are a low-mass core (NGC 1333 IRAS 4), three high-mass cores spanning a range of star-forming environments and evolutionary states (W49, AFGL 2591, and IRAS 20126), and a photodissociation region (the Orion Bar). The SLS will use the unique spectral imaging capabilities of HARP-B/ACSIS (Heterodyne Array Receiver Programme B/AutoCorrelation Spectrometer and Imaging System) to study the molecular inventory and the physical structure of these objects, which span different evolutionary stages and physical environments and to probe their evolution during the star formation process. As its name suggests, the SLS will provide a lasting data legacy from the JCMT that is intended to benefit the entire astronomical community. As such, the entire data set (including calibrated spectral data cubes, maps of molecular emission, line identifications, and calculations of the gas temperature and column density) will be publicly available.
\end{abstract}

Online material: color figure

\section{INTRODUCTION}

In early 2006, the James Clerk Maxwell Telescope (JCMT) on the summit of Mauna Kea, Hawaii, began installation of a new generation of scientific instruments: SCUBA-2 and HARPB/ACSIS (Holland et al. 2006; Smith et al. 2003; Buckle et al. 2007), which will revolutionize our understanding of the submillimeter sky. HARP-B (Heterodyne Array Receiver Pro-

\footnotetext{
${ }^{1}$ Department of Physics and Astronomy, University of Calgary, Calgary, AB, Canada; plume@ism.ucalgary.ca.

${ }^{2}$ School of Physics and Astronomy, University of Manchester, Manchester, UK; gary.a.fuller@manchester.ac.uk.

${ }^{3}$ SRON Netherlands Institute for Space Research, Groningen, Netherlands; f.p.helmich@sron.rug.nl.

${ }^{4}$ Kapteyn Astronomical Institute, University of Groningen, Groningen, Netherlands.

${ }^{5}$ School of Mathematics and Physics, Queen's University Belfast, Belfast, Northern Ireland.

${ }^{6}$ Department of Physics and Astronomy, University College London, London, UK.

${ }^{7}$ Cavendish Laboratory, Cambridge, UK.

${ }^{8}$ Joint Astronomy Centre, Hilo, HI.

9 CESR-UPS, Centre National de la Recherche Scientifique, Toulouse, France.
}

gramme B) is a 16 element heterodyne array receiver (in a $4 \times 4$ pixel configuration with a $2^{\prime} \times 2^{\prime}$ field of view) designed to operate between 325 and $375 \mathrm{GHz}$, with a receiver noise temperature of less than $330 \mathrm{~K}$ (single-sideband [SSB]) averaged across the array. HARP-B was built by the Mullard Radio Astronomy Observatory (MRAO) at the University of Cam-

\footnotetext{
${ }^{10}$ Laboratoire d'Astrophysique de l'Observatoire de Grenoble, Grenoble, France.

${ }^{11}$ Leiden Observatory, Leiden University, Leiden, Netherlands.

${ }^{12}$ Department of Physics and Astronomy, University of British Columbia, Vancouver, BC, Canada.

${ }^{13}$ University of Exeter, UK.

${ }^{14}$ Herzberg Institute of Astrophysics/National Research Council of Canada, Victoria, BC, Canada.

${ }^{15}$ St. Mary's University, Halifax, NS, Canada.

16 Astrophysics Research Institute, Liverpool John Moores University, Birkenhead, UK.

${ }^{17}$ Institute of Physics I, University of Köln, Köln, Germany.

${ }^{18}$ Max-Planck-Institut, Bonn, Germany.

${ }^{19}$ NASA Ames Research Center, Moffett Field, CA.

${ }^{20}$ Centre for Astrophysics Research, Science and Technology Research Institute, University of Hertfordshire, Hatfield, UK.

${ }^{21}$ The Open University, Milton Keynes, UK.

${ }^{22}$ CCLRC Rutherford Appleton Laboratory, Didcot Oxfordshire, UK.
} 
bridge (UK), in collaboration with the Joint Astronomy Centre (Hawaii), the Astronomy Technology Centre at the Royal Observatory in Edinburgh (UK), and the Herzberg Institute of Astrophysics in Victoria (Canada). The SIS (superconductorinsulator-superconductor) junctions at the heart of HARP-B were provided by the Delft Institute of Microelectronics and Submicron Technology (DIMES) in the Netherlands. HARP$\mathrm{B}$ is designed to work with the JCMT's new spectrometer: the Auto-Correlation Spectrometer and Imaging System (ACSIS). ACSIS was built by the Dominion Radio Astronomy Observatory in Penticton, British Columbia (Canada), and the Astronomy Technology Centre at the Royal Observatory, Edinburgh (UK), and allows 16 simultaneous intermediatefrequency inputs with a $2 \mathrm{GHz}$ bandwidth.

While SCUBA-2 will allow astronomers to quickly map the continuum emission at submillimeter wavelengths and thus examine the nature and distribution of the cold dust, HARP-B/ ACSIS will allow them to efficiently map the molecular gas. Molecular emission lines can provide direct information on the physical conditions (density, temperature, pressure, and optical depth) and kinematics of the emitting gas. Given the high speed and sensitivity of HARP-B/ACSIS, combined with the largest aperture telescope at an excellent observing site, there will be no comparable instrument for mapping large areas of the sky at submillimeter wavelengths or for surveying molecular emission across the entire $345 \mathrm{GHz}$ band.

Large-scale, well-coordinated surveys were called for to fully exploit these exciting new instruments and, in response, the JCMT Legacy Surveys were born. These surveys are ambitious, community-driven projects involving over 100 astronomers from Canada, the UK, the Netherlands, and a few additional nonpartner countries. This paper describes the goals of the JCMT Spectral Legacy Survey (SLS), one of the seven Legacy Surveys approved by the JCMT Board of Directors following a rigorous peer-review process. In a $2 \mathrm{yr}$ period beginning in 2007, the SLS will receive $187 \mathrm{hr}$ of band 4 weather conditions $\left(0.12<\tau_{225 \mathrm{GHz}}<0.2\right)$, in order to survey the molecular emission of a variety of star-forming regions. The SLS will provide a lasting data legacy from the JCMT that is intended to benefit the entire astronomical community by fully exploiting the spectral imaging capabilities of HARP-B. The SLS is led by coordinators from the three partner countries: G. Fuller (principal investigator) for the UK, R. Plume for Canada, and F. Helmich for the Netherlands.

\subsection{Scientific Background}

It is well known that stars form in molecular clouds, and most stars form in clusters in giant molecular cloud (GMC) cores. Although this statement is commonly accepted, the details of the process are not well known. For both low-mass and high-mass stars, several phases in the star formation process have been recognized (e.g., Shu et al. 1987; McKee \& Tan 2002). These include, first, a precollapse cloud-core phase char- acterized by low temperatures; second, a deeply embedded phase in which a cool, protostellar object is surrounded by warm gas, an infalling envelope, and stellar winds; and third, later phases, in which strong stellar winds have progressively cleared the immediate environment and exposed the newly formed star and its surrounding protoplanetary disk.

Although complicated, it is possible to build a physical picture of evolving, star-forming cores. Since these cores are extended objects that also contain small-scale structures, we will require detailed observations sensitive to a range of size scales and physical conditions. Interferometers can provide the spatial distribution of dust and gas at the smallest size scales, while single-dish telescopes equipped with large-format, widebandwidth detectors are capable of simultaneously imaging a large number of molecules (albeit at lower spatial resolution). With proper physical modeling, the combination of the two leads to a better understanding of the physics of cores and therefore helps to constrain the formation mechanism of stars and stellar clusters. For example, van der Tak et al. (2000) have revealed the detailed physical structure of a dozen regions of high-mass star formation, including AFGL 2591, using a combination of single-dish and interferometer observations, which made it possible to assign different velocity and excitation components to different physical parts of the core.

The dynamical and physical evolution during star formation is also accompanied by a strong chemical evolution. For example, the cold cloud-core phase is dominated by relatively simple molecular species. In contrast, the warm gas around protostellar objects is characterized by surprisingly complex species. This chemical evolution points toward the interplay of a multitude of chemical processes, including ion-molecule reactions at low temperatures in dark cloud cores, accretion and reaction on cold dust grains, and evaporation once the newly formed star turns on (e.g., van Dishoeck \& Blake 1998). While the composition, origin, and evolution of interstellar molecules in regions of star formation are important topics in their own right, observations of molecules can also provide direct information on the physical conditions of the emitting gas and hence of the physical evolution of a star-forming region. The large number of rotational lines from low-lying levels of a species and its isotopologues - each with its own collisional and radiative excitation rates-probe the density, temperature, and optical depth of gas very effectively over a wide range of conditions relevant for regions of star formation. Furthermore, these molecules play an important role in regulating the temperature, and hence pressure, via the process of heating and cooling through line absorption and emission.

To date, about 151 different molecular species have been detected in interstellar and circumstellar gas (Cologne Database for Molecular Spectroscopy ${ }^{23}$ ), but few of these species have been studied in more than a few sources. Our understanding

\footnotetext{
${ }^{23}$ See http://www.cdms.de.
} 
of the molecular inventory of sources and its distribution is poor: few sources have been systematically surveyed at any frequency band, and most such surveys have been limited to a single position toward a source. None to date have imaged every molecular line (see Blake et al. 1996). However, regions of star formation are complex by nature. Stars rarely form in isolation: protostars often form in binary systems that are themselves in groups or clusters. Furthermore, star formation generally involves simultaneous infall and outflow, circumstellar disks, and the presence of regions of warm, dense gas (hot cores) near the protostar itself. Each of these regions is characterized by its own density, temperature, and velocity structure, as well as its unique chemical composition. Fully disentangling this spatial complexity requires mapping in a large number of transitions of a variety of molecules.

The study of the formation of high-mass stars is especially complicated by the fact that chemistry timescales (and therefore the timescale for cooling through line emission) are comparable to the accretion timescale. In addition, the large density and temperature gradients involved complicate the physical modeling. Therefore, detailed observations are key to understanding the processes involved in creating the stellar clusters that shape the Galaxy. Over the last few decades, there have been a few studies of the chemical composition of prominent star-forming regions such as Orion (Leurini et al. 2006; Comito et al. 2005; White et al. 2003; Schilke et al. 1997, 2001; Blake et al. 1987; Sutton et al. 1991), Sgr B2 (Nummelin et al. 1998; Goicoechea et al. 2004), and W3 (Helmich \& van Dishoeck 1997), the most infrared-bright star-forming region in the sky. However, only recently have spectral surveys of less luminous sources become feasible. In general, being less luminous implies that the source is more deeply embedded and thus in an earlier evolutionary stage where outflows have not yet cleared away the molecular environment. For example, the results of the spectral survey of IRAS 16293-2422 (initiated by the results of Cazaux et al. [2003] and shown in its first results by Parise et al. [2005] on HDO) enable us to probe the chemistry in an unprecedented way and extend our knowledge to earlier stages of star formation.

The complete molecular inventory and thus the physical, chemical, and evolutionary status of sources are only fully accessible through unbiased spectral surveys. The factor of 16 increase in mapping speed provided by HARP-B together with the spectral coverage and resolution of ACSIS provide the most potent instrument available to obtain the molecular inventory of sources in the $345 \mathrm{GHz}$ band.

The $325-373 \mathrm{GHz}$ spectral range is rich in known molecular transitions: Lovas (2004) lists 866 transitions arising from 82 different molecular species. Many of the transitions in this band have a range of excitation energies from tens to hundreds of kelvin, making this window particularly useful for studying material that is heated above the $\approx 10 \mathrm{~K}$ of ambient molecular gas in clouds, and for constraining the physical and chemical structure on scales smaller than the beam. However, this band has not been thoroughly explored in a variety of different sources, even though it is particularly important in the future of submillimeter astrophysics; it corresponds to the Atacama Large Millimeter Array (ALMA) frequency 7 band, which the ALMA Design Reference Science Plan suggests will be used for more than $30 \%$ of the ALMA observing in all areas of science.

\section{SCIENCE GOALS}

The goal of the SLS is to use molecular line observations to help understand the evolution of young stellar objects spanning a range of evolutionary stages and environmental conditions. This will be achieved by obtaining a complete molecular line inventory in three different types of sources: low-mass protostars, young high-mass sources, and photodissociation regions (PDRs). Combining these data with detailed chemical and physical models of the sources, the survey aims to improve our understanding of molecular tracers as probes of the astrophysics and evolution of sources. In particular, this survey will:

1. Provide an inventory of the column densities and spatial distributions of the molecular species toward sources of different types and age.

2. Provide a broad-based derivation of the physical and chemical conditions (i.e., temperature, density, velocity, and chemical structure) of the sources on scales both smaller and larger than that of the primary beam.

3. Identify important chemical diagnostics of the physical and chemical processes occurring in different types of sources and different evolutionary states.

Beyond this, the survey will provide a uniform data set for future explorations of these sources to the general astronomical community. In conjunction with similar, higher frequency surveys being planned for the Herschel Space Observatory, it will also help us determine what constitutes a typical spectrum in sources with different environmental and evolutionary states.

\subsection{Molecular Inventory}

Emission features in each source will be identified using the Jet Propulsion Laboratory, ${ }^{24}$ Cologne (see footnote 23), and other available line catalogs. Tools for this type of analysis (e.g., CASSIS ${ }^{25}$; XCLASS, P. Schilke 2006, private communication) are currently under development. The spatial information and the different line widths likely to be seen at different positions will be used to aid in the identification of blended or confused emission features. Unidentified features will be examined to determine whether they are consistent with being undocumented transitions of other species present in the spectra. The line velocity and frequency, intensity, integrated intensity, and width will be determined initially through Gaussian

\footnotetext{
${ }^{24}$ See http://spec.jpl.nasa.gov.

${ }^{25}$ See http://pc-126.cesr.fr.
} 
fitting and/or a one-dimensional clumping analysis, and then cataloged.

The unique capability of HARP-B/ACSIS to simultaneously map sources spatially and spectrally will be of key importance in disentangling the spatial complexity of sources and unraveling the contribution of the different components of the environments (outflows, infalling material, disk, and quiescent cloud). Specifically, we will use the images, line widths, and excitation of the different species to identify the signatures of these different components so as to be able to separately determine their physical properties. Column densities will then be calculated from the line-integrated intensities initially using rotation diagrams or statistical equilibrium modeling (Schöier et al. 2005). We will derive column densities for the molecules with the brightest and greatest number of transitions in the survey. Ultimately, it will be a goal to calculate the spatial abundance structure for the majority of the detected species, although this will be more difficult and may require additional observations of lines at other frequencies for molecules with relatively few transitions in the SLS survey band.

Apart from the numerous lines we expect to identify through the SLS, we also expect to find a number of lines that we will be unable to identify. These "U lines" will be flagged for future analysis.

\subsection{Physical Conditions and Abundance Profiles}

Millimeter and submillimeter continuum observations will be used to determine the temperature and density distribution for each source (after correcting the continuum flux for contamination by the line emission). For some of the sources, such data already exist; for others, these will be obtained during other observations. Initially, the modeling will be one-dimensional in order to rapidly estimate the molecular column densities and distributions. However, one-dimensional models are unlikely to adequately reproduce the observations, so we plan to extend the modeling to two dimensions and, if possible, to three dimensions.

Molecular line observations can also provide information about the physical conditions in these sources. The most obvious of these is the use of $\mathrm{CO}$ to trace the structure of the bulk of the gas in molecular clouds and the small masses of gas at high velocities in outflows from young stars. Rotation diagram analyses of various molecules will also be used to determine the kinetic temperatures and column density of the gas. Furthermore, for molecules that have known collision rates, large velocity gradient codes can also be used to determine the gas densities, column densities, and temperatures.

With the density and temperature structure determined, the emission expected for different abundances and distributions of the molecular species will be modeled using radiative transfer models such as RATRAN (Hogerheijde \& van der Tak 2000) to determine which best reproduce the observed spectra. Initially, this will be done by adopting trial abundance profiles assuming constant abundance, jump, antijump, or drop abundance profiles (Schöier et al. 2004). Models of the chemistry of these sources will then be generated to explain the origin of these distributions of species and to investigate the evolution of sources (see Fig. 1 of Doty et al. [2004] for a useful graphical explanation of the entire modeling procedure). The best-fit models will be used to generate and explore the characteristic, or template, broadband spectra resulting from the different physical environments in the sources. These spectra will provide a means of decomposing the broadband $345 \mathrm{GHz}$ spectra of other sources in such a way as to identify the various physical and chemical processes taking place in the sources.

\subsection{Chemistry as a Probe of Astrophysics and Evolution}

Rarer species are important tracers of specific environments in clouds or embedded sources. Examples include the chemistry of deuterium-containing species, sulfur species, and complex organic molecules.

1. Deuterium chemistry.-If fractionation did not take place, a species containing deuterium would be $>10^{5}$ times less abundant than its hydrogen-bearing counterpart and thus would be completely undetectable. However, in cold $(T<20 \mathrm{~K})$ regions, the zero-point energy difference between a hydrogen-containing species and its deuterium-substituted isotopologue leads to enhanced abundances of the deuterium-containing species (i.e., above the cosmic $\mathrm{D}$ to $\mathrm{H}$ ratio). Recent work on deuterated species has shown that in dense, cold regions their abundance is enhanced even further, since the species that would otherwise destroy the deuterated molecules freeze out onto dust grains. Thus, deuterated molecular species uniquely probe extremely cold, dense material. For example, the deuterated form of a principal ion in molecular clouds, $\mathrm{H}_{2} \mathrm{D}^{+}$, is very weak $(0.1 \mathrm{~K})$ toward Class 0 objects (Stark et al. 1999), but has a $1 \mathrm{~K}$ line toward a cold, dense, quiescent prestellar core where $\mathrm{CO}$ is heavily depleted (Caselli et al. 2003). Clearly mapping such a line around an embedded protostar will directly trace the densest, coldest circumstellar material as yet unaffected by the protostar.

Conversely, deuterated neutral species are also observed toward several hot cores (e.g., Hatchell et al. 1999 and references therein; Pardo et al. 2001; Osamura et al. 2004). Since the gas temperatures in these regions exceed $50 \mathrm{~K}$ and are often $>100 \mathrm{~K}$ (Hatchell et al. 1998a), these species could not have achieved such high fractionations in their current environment. The most likely explanation is that they have evaporated from material previously frozen onto grains as the grains are heated by the protostar (Rodgers \& Millar 1996). The high deuteration in hot cores points to these grain mantles having been formed when cold gas, $T<50 \mathrm{~K}$, condensed onto the grains prior to the formation of, and heating by, an embedded protostar.

2. Sulfur species.-The release of species from grain mantles as the grains are heated injects new species into the gas phase 
that can be directly detected, but which also drive new gasphase reactions. One such reactant released when grain mantles evaporate is sulfur (Charnley 1997). It may be possible to use the subsequent sequential formation of sulfur-bearing daughter species as a chemical clock measuring the time since the mantles were injected, an idea that has since been investigated not only for high-mass sources (Hatchell et al. 1998b; van der Tak et al. 2003) but also for lower luminosity sources (Buckle \& Fuller 2003), with more recent modeling work being done by Wakelam et al. (2004). Observations, particularly imaging, of the multiple daughter species in a particular chemical network are vital for determining the nature of the chemical pathways involved (Jørgensen et al. 2004). The SLS will be in a unique position to investigate this issue.

3. Complex organic species.-These molecules appear in the spectra of both low-mass (e.g., Bottinelli et al. 2004; Jørgensen et al. 2005) and high-mass star-forming regions (e.g., Comito et al. 2005) and, in both, may be used as chemical clocks to indicate source ages and evolutionary status. In prestellar cores, the organics are very unsaturated and consist of carbon chains such as the cyanopolyynes $\left(\mathrm{HC}_{2 n+1} \mathrm{~N}, n=1-5\right)$, whereas in high-mass regions the organics are usually highly saturated and very enhanced in abundance, e.g., methanol, ethanol, $\mathrm{C}_{2} \mathrm{H}_{5} \mathrm{OH}$, and dimethyl ether $\left(\mathrm{CH}_{3} \mathrm{OCH}_{3}\right)$. In low-mass regions, the carbon chains are abundant at early times because the conversion of carbon to $\mathrm{CO}$ has not yet gone to completion. In high-mass regions, the saturated organics arise as both the direct products (parents) of grain ice evaporation and as the daughter species that result from a high-temperature chemistry involving these parents. Nomura \& Millar (2004) have shown how the spatial distributions of molecules in hot core sources can be used to probe the chemical evolution of the hot gas, the ice composition of the grains, and the density and temperature profiles of the warm gas. It was thought that low-mass star-forming regions had a different chemical evolution after the formation of a central protostellar source, but the recent detection of complex saturated organic molecules in IRAS 16293-2422 (Cazaux et al. 2003) — in a so-called hot corino - has led to a reevaluation; it is now thought that these regions may also show the effects of grain evaporation and subsequent chemical evolution, albeit at somewhat lower temperatures and densities than in highmass hot cores. A sensitive search for organics will allow us to probe the cloud history, in particular, the nature of the material frozen on ices, the influence of the central protostar in warming its surrounding natal cloud, the influence of gas temperature on the subsequent processing of the gas, and the timescales over which this processing has been occurring.

\subsection{Wider Applications}

The SLS will be a crucial pathfinder experiment for spectroscopic studies of star-forming regions, by identifying the best combinations of spectral lines to observe in different types of sources. Thus, the SLS sources will serve as templates for observations using next-generation instruments such as the extended Submillimeter Array, ALMA, and Herschel.

However, in addition to providing a basis for understanding the overall molecular emission from sources in molecular clouds in our galaxy, the results from the SLS will be important in understanding more distant sources. An example of this can be seen in the recent work on NGC 253 by Martín et al. (2005), in which observations of sulfur-containing species are compared with galactic regions to determine the state of the molecular clouds in the galaxy. Another example is the inference that widespread HCO emission in M82 is the result of the illumination of its molecular clouds by a strong radiation field (García-Burillo et al. 2002).

A further application of complete spectral scans is related to understanding the continuum emission from sources. For sources with strong spectral lines, the lines can become important contributors to the broadband flux. For example, toward Orion, as much as $60 \%$ of the broadband $345 \mathrm{GHz}$ flux could be due to lines in the band, not due to continuum emission from dust (White et al. 2003; Groesbeck 1995). In particular, toward Orion $\mathrm{KL}$, the major contaminants are the multitude of lines of $\mathrm{SO}_{2}$ and $\mathrm{SO}, \mathrm{CO}$ being only the third most important species (Schilke et al. 1997). However, the dominance of $\mathrm{SO}_{2}$ as a contaminant of the continuum emission is not universal. Thompson \& Macdonald (1999) found that in G5.89-0.39, CO was the dominant species, possibly due to increased beam dilution for $\mathrm{SO}_{2}$. The fully sampled maps provided by the SLS will be able to determine the spatial distribution of the various molecular species and thus identify the underlying cause for differences in the $\mathrm{SO}_{2} / \mathrm{CO}$ line ratios.

Regardless of the source of contamination, an accurate determination of the temperature and density profile from a source requires the measured broadband flux to be corrected for contamination, something that can only be done with complete spectral scans.

\section{TARGET SOURCES}

We will target sources that have been previously studied at other wavelengths in order to provide continuity with existing studies. All the objects have also been selected as targets (or potential targets) for the Herschel Heterodyne Instrument for the Far-Infrared (HIFI) spectral survey. The SLS observations will consist of a fully sampled HARP-B footprint centered on each target source to determine its molecular inventory. The desired spectral resolution is $1 \mathrm{~km} \mathrm{~s}^{-1}$. This combined spatial and spectral information will, for the first time in a spectral survey, allow us to directly determine molecular abundances not only toward each target, but also in their immediate environment. The sources to be observed are described below. 


\subsection{Low-Mass Source}

For the low-mass protostars, we will observe the well-studied source NGC 1333 IRAS 4. Located in the L1430 core of Perseus, NGC 1333 ( $d \sim 320 \mathrm{pc}$; de Zeeuw et al. 1999) is a region forming many young stellar objects (YSOs). IRAS 4 is interesting, as it is one of the first and one of the youngest (André et al. 1993) protobinaries ever detected. IRAS $4 \mathrm{~A}$ and $4 \mathrm{~B}$ are separated by a distance of $30^{\prime \prime}$, and recent evidence has shown that these sources are binaries themselves. IRAS 4A has been resolved into two sources (4A and $4 \mathrm{~A}^{\prime}$ ) separated by $2^{\prime \prime}$ (Lay et al. 1995), and IRAS 4B has been resolved into 4B and 4B', with a 10" separation (Looney et al. 2000). IRAS 4B may in fact be a multiple system itself, with individual YSOs separated by $0.5^{\prime \prime}$. Both IRAS $4 \mathrm{~A}$ and $4 \mathrm{~B}$ are associated with molecular outflows (Blake et al. 1995; Choi 2001, 2005). That from $4 \mathrm{~A}$ is highly collimated in the northeast-southwest direction, although the northeast lobe shows a sharp bend, interpreted by Choi (2005) as evidence for a jet-core collision. The outflow from IRAS 4B, in contrast, is quite compact. Observations and modeling of P Cygni profiles in IRAS 4A and 4B have led to YSO masses of 0.71 and $0.23 M_{\odot}$ and accretion rates of $1.1 \times 10^{-4}$ and $3.7 \times 10^{-5} M_{\odot} \mathrm{yr}^{-1}$, respectively (Di Francesco et al. 2001). The resultant accretion timescales place the objects at $\sim 6500 \mathrm{yr}$ old. A number of important molecular species, including some complex molecules (Bottinelli et al. 2004), have been observed at single positions in these two sources (Jørgensen et al. 2004, 2005; Maret et al. 2004). These observations have shown some interesting chemical differences between IRAS 4A and 4B. In $4 \mathrm{~A}$, the data are well fit by abundance drops of a factor of 10 100 in the dense $\left(n>6 \times 10^{5} \mathrm{~cm}^{-3}\right)$, cold $(T<40 \mathrm{~K})$ regions of the envelope. In contrast, in $4 \mathrm{~B}$, the observations can be explained by an abundance increase in the compact outflow where the temperature rises above $30 \mathrm{~K}$, and not from the extended envelope. HARP-B maps of these two sources will allow us to examine the extended environments of both sources in better detail.

Both IRAS 4A and 4B will be covered in a single, fully sampled HARP-B footprint. The choice of $1 \mathrm{~km} \mathrm{~s}^{-1}$ channels is a compromise between a desire to spectrally resolve lines, covering the frequency band in as few settings as possible, and the required noise level. Although molecular lines from the most quiescent regions around a low-mass protostar may have full width at half-maxima of less than $1 \mathrm{~km} \mathrm{~s}^{-1}$, observations of IRAS 16293-2422 indicate that toward this source a typical line has a width of 1.5-2 $\mathrm{km} \mathrm{s}^{-1}$ (van Dishoeck et al. 1995; Ceccarelli et al. 2000). The required noise level is set by existing observations of IRAS 16293, in which complex organics tracing the hot gas around the star are detected at a level of $\sim 0.05 \mathrm{~K}$, implying the need to reach a noise level of $0.015 \mathrm{~K}$ to detect such lines with a peak signal-to-noise ratio of 3 . This low noise level will also act to alleviate some of the possible bandwidth dilution of any narrow lines from the most quiescent gas.

\subsection{High-Mass Sources}

For the high-mass sources, we will observe W49, AFGL 2591, and IRAS 20126, which are believed to span a range of star-forming environments and evolutionary states. The lines toward these high-mass objects typically have widths of significantly greater than $2 \mathrm{~km} \mathrm{~s}^{-1}$ (Macdonald et al. 1996; Thompson \& Macdonald 1999), so the choice of $1 \mathrm{~km} \mathrm{~s}^{-1}$ resolution was determined by the maximum bandwidth with ACSIS. An optically thick line from a 1 " diameter hot core with a temperature of $100 \mathrm{~K}$ toward one of these sources will have a measured line peak temperature of $0.4 \mathrm{~K}$ in the JCMT beam. To be able to detect the optically thin lines that provide most of the information on the sources will require reaching noise levels considerably less than this. We intend to reach a noise level of $0.04 \mathrm{~K}$ per beam in $1 \mathrm{~km} \mathrm{~s}^{-1}$ channels that will be smoothed to $2.5 \mathrm{~km} \mathrm{~s}^{-1}$ channels, giving a noise level of $0.025 \mathrm{~K}$. This noise level is lower than that for any of the existing surveys in this band and so will probe the chemistry of these regions to a somewhat better depth.

\subsubsection{W49}

W49A was chosen as an example of an extremely active star-forming region (e.g., Homeier \& Alves 2005). Evidence of this comes from the fact that W49A is associated with one of the most powerful $\mathrm{H}_{2} \mathrm{O}$ masers in the Galaxy (Genzel et al. 1978 ) and that it is also one of the most luminous regions in the Galaxy ( $L_{\text {bol }} \sim 10^{7} L_{\odot}$; Ward-Thompson \& Robson 1990). W49 is a distant ( $d=11.4 \mathrm{kpc}$; Gwinn et al. 1992) molecular cloud complex consisting of two distinct regions: a supernova remnant (W49B) and a giant $\mathrm{H}$ II region (W49A), separated by $\sim 12^{\prime}$. W49A is composed of a number of optically obscured, compact H II regions-W49SW, W49SE, and, the strongest, W49NW (more commonly named W49N; Harvey et al. 1977) surrounded by a massive molecular cloud $\left(M>10^{5} M_{\odot}\right.$; Mufson \& Liszt 1977; Simon et al. 2001). Spectrally, W49A is extremely complex, containing numerous features contributed by W49A itself, as well as additional clouds associated with the Sagittarius spiral arm, which crosses the line of sight twice. W49A and the associated line-of-sight clouds have been the subject of numerous spectroscopic studies (both emission and absorption) involving species such as $\mathrm{H}$ I (Radhakrishnan et al. 1972), $\mathrm{HCO}^{+}$and $\mathrm{HCN}$ (Nyman 1983), OH (Cohen \& Willson 1981), $\mathrm{H}_{2} \mathrm{CO}$ (Mufson \& Liszt 1977; Bieging et al. 1982), CO (Mufson \& Liszt 1977), SiO (Lucas \& Liszt 2000), CS (Greaves \& Williams 1994), and $\mathrm{H}_{2} \mathrm{O}$ (Plume et al. 2004).

\subsubsection{AFGL 2591}

The bright infrared source AFGL $2591\left(L>20,000 L_{\odot}\right)$ is a prototypical object in which to study physical and chemical 


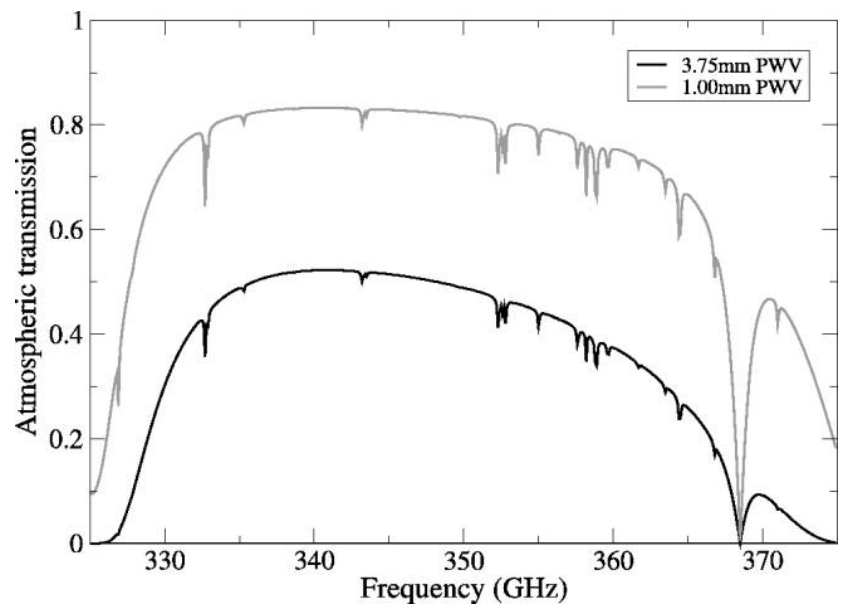

Fig. 1.-Atmospheric transmission as a function of frequency for the $345 \mathrm{GHz}$ window. The upper, gray line corresponds to a good night on Mauna Kea (i.e., band 1 weather), with $1 \mathrm{~mm}$ of PWV, or $\tau_{225 \mathrm{GHz}} \approx 0.05$. The lower, black line indicates the band 4 weather in which the SLS will observe, corresponding to $3.75 \mathrm{~mm} \mathrm{PWV}$, or $\tau_{225 \mathrm{GHz}} \approx 0.16$. [See the electronic edition of the PASP for a color version of this figure.]

processes during high-mass star formation. Located in the Cygnus $\mathrm{X}$ region, recent observations place it at a distance of $1.7 \mathrm{kpc}$ (Schneider et al. 2006). Existing JCMT observations (e.g., van der Tak et al. 1999; Doty et al. 2002) show a rich molecular line spectrum, which, coupled with its proximity, makes AFGL 2591 a prime target for the SLS project. In addition, its physical structure is well characterized. The temperature and density structure of the envelope are well known from the work by van der Tak et al. (1999) and, using this model, the chemical structure has also been investigated in detail (Doty et al. 2002). A powerful molecular outflow is visible in high-velocity mid-infrared $\mathrm{CO}$ absorption lines, as well as large lobes of millimeter-wave emission (Mitchell et al. 1990, 1992). High angular resolution mapping of $\mathrm{H}_{2} \mathrm{O}$ $22 \mathrm{GHz}$ masers suggests precessing motions in the outflow (Trinidad et al. 2003). The detection of $\mathrm{CO}^{+}$and $\mathrm{SO}^{+}$by Stäuber et al. (2006) suggests that X-ray ionization plays a role for the inner envelope. Interferometer observations of the $\mathrm{H}_{2}^{18} \mathrm{O} 203 \mathrm{GHz}$ line reveal a flattened structure with a velocity gradient, possibly a massive circumstellar disk (van der Tak et al. 2006).

\subsubsection{IRAS $20126+4104$}

IRAS $20126+4104$ is another YSO located in the Cygnus $\mathrm{X}$ region, thus providing us with two objects that formed at the same distance and, likely, in similar environments. Its luminosity is somewhat lower than that of AFGL 2591 ( $L \sim$ $10^{4} L_{\odot}$ ), possibly suggesting a younger evolutionary state. Recent observations by Cesaroni et al. (2005) suggest that the YSO mass is $\sim 7 M_{\odot}$, embedded in a surrounding 0.4 pc diameter core whose mass is $230 M_{\odot}$ (Estalella et al. 1993).
United Kingdom Infrared Telescope (UKIRT) images in $K$ band further suggest that the central object may in fact be a binary system (Sridharan et al. 2005). IRAS 20126 has been known for some time to contain a powerful molecular outflow oriented northwest-southeast, which appears to be bounded by an Sshaped distribution of $\mathrm{H}_{2}$ emission knots (Shepherd et al. 2000; Lebrón et al. 2006). The distribution of the $\mathrm{H}_{2}$ emission knots has led to the suggestion that the molecular jet in IRAS 20126 is precessing (Shepherd et al. 2000; Cesaroni et al. 2005; Lebrón et al. 2006). Observations of $C^{34} S$ by Cesaroni et al. (2005) have confirmed the presence of a large Keplerian disk with a radius of $5000 \mathrm{AU}$. However, this disk is thought to be too large to drive the observed outflow. The observations of Sridharan et al. (2005), however, suggest the presence of a more compact disk $(R \sim 1000 \mathrm{AU})$, which may be more directly related to the outflow.

\subsection{Photodissociation Regions}

Based on existing observations and modeling of PDRs, we have selected the Orion Bar region as our target PDR. This region is subject to a very high UV flux, $\sim 5 \times 10^{4}$ times greater than the typical interstellar field, and is thus likely to clearly show the effects of the radiation on the molecular gas. Previous molecular line observations (Leurini et al. 2006; Hogerheijde et al. 1995; Jansen et al. 1995) have shown dramatic abundance variations across the bar. These observations highlight the importance of having combined spatial and spectral information to disentangle complex abundance variations. Line widths of 1.8-4 $\mathrm{km} \mathrm{s}^{-1}$ have been observed toward a number of PDR sources by Schilke et al. (2001). Reaching the same noise level as toward the high-mass protostars would allow us to detect even species as rare as $\mathrm{HOC}^{+}$at a greater than $4 \sigma$ level (Savage \& Ziurys 2004).

\section{OBSERVING STRATEGY}

Figure 1 shows the atmospheric transmission over the $345 \mathrm{GHz}$ window. Given the poor transmission at the edges of the window (i.e., below $330 \mathrm{GHz}$ and above $360 \mathrm{GHz}$ ), observations at these frequencies would be prohibitively time consuming. Thus, the SLS will only cover 330-360 GHz. To cover this bandpass will require 22 tunings of the receiver (although ACSIS will allow a $2 \mathrm{GHz}$ bandwidth, the maximum bandwidth per mixer from HARP-B is $\sim 1.8 \mathrm{GHz}$ ), with each consecutive frequency tuning overlapping by $10 \%$ of the bandwidth to avoid gaps in the spectra and to provide consistency between the tunings. Since HARP-B is a SSB instrument, larger overlaps are not required, since there is no need to deconvolve emission from different sidebands. Table 1 gives the frequency settings and integration times required to achieve uniform rms noise levels. These values assume grade 4 weather conditions (i.e., $3.75 \mathrm{~mm}$ precipitable water vapor [PWV]), a source elevation of $90^{\circ}$, and a fully sampled HARP-B footprint.

Using ACSIS with a $2 \mathrm{GHz}$ bandwidth will give $0.977 \mathrm{MHz}$ 
TABLE 1

SLS Frequencies and Integration Times (Assuming $90^{\circ}$ Source Elevation)

\begin{tabular}{|c|c|c|}
\hline $\begin{array}{l}\text { Frequency } \\
(\mathrm{GHz})\end{array}$ & $\begin{array}{l}\text { Low-Mass Sources } \\
\left(T_{\mathrm{rms}}=0.03 \mathrm{~K}^{\mathrm{a}}\right) \\
(\mathrm{hr})\end{array}$ & $\begin{array}{c}\text { High-Mass Sources } \\
\left(T_{\mathrm{rms}}=0.08 \mathrm{~K}^{\mathrm{a}}\right) \\
(\mathrm{hr})\end{array}$ \\
\hline $332.237 \ldots \ldots$ & 6.62 & 0.93 \\
\hline $333.553 \ldots \ldots$ & 5.51 & 0.77 \\
\hline $334.868 \ldots \ldots$ & 4.94 & 0.69 \\
\hline $336.184 \ldots \ldots$ & 4.61 & 0.65 \\
\hline $337.500 \ldots \ldots$ & 4.40 & 0.62 \\
\hline $338.816 \ldots \ldots$ & 4.29 & 0.60 \\
\hline $340.132 \ldots \ldots$ & 4.22 & 0.59 \\
\hline $341.447 \ldots \ldots$ & 4.20 & 0.59 \\
\hline $342.763 \ldots \ldots$ & 4.22 & 0.59 \\
\hline $344.079 \ldots \ldots$ & 4.25 & 0.60 \\
\hline $345.395 \ldots \ldots$ & 4.30 & 0.60 \\
\hline $346.711 \ldots \ldots$ & 4.38 & 0.62 \\
\hline $348.026 \ldots \ldots$ & 4.48 & 0.63 \\
\hline $349.342 \ldots \ldots$ & 4.61 & 0.65 \\
\hline $350.658 \ldots \ldots$ & 4.77 & 0.67 \\
\hline $351.974 \ldots \ldots$ & 5.04 & 0.71 \\
\hline $353.289 \ldots \ldots$ & 5.25 & 0.74 \\
\hline $354.605 \ldots \ldots$ & 5.50 & 0.77 \\
\hline $355.921 \ldots \ldots$ & 5.83 & 0.82 \\
\hline $357.237 \ldots \ldots$ & 6.33 & 0.89 \\
\hline $358.553 \ldots \ldots$ & 7.14 & 1.00 \\
\hline $359.868 \ldots \ldots$ & 7.77 & 1.09 \\
\hline
\end{tabular}

${ }^{\mathrm{a}}$ Assuming a velocity resolution of $2.5 \mathrm{~km} \mathrm{~s}^{-1}$.

wide channels. Since for all the sources except NGC 1333 IRAS 4 the expected line widths are greater than this, for much of the analysis the data will be rebinned to $2.5 \mathrm{~km} \mathrm{~s}^{-1}$ channels, which is also the channel width used in previous single-position surveys at this frequency band. The survey will aim to observe similar frequency coverage for all the sources.

Table 2 lists the center positions for the fields for each source, as well as the estimated total integration times required in the band 4 weather conditions $\left(0.12<\tau_{225 \mathrm{GHz}}<0.2\right)$ allocated to the SLS, given the average elevation of the sources.

\subsection{Data Products Provided to the Community}

The goal of the SLS is to provide to the astronomical community a uniform and unbiased spectral survey of a variety of star-forming regions. As such, the full spectral data cubes will be publicly available after a 1 year proprietary period. Thus, we intend to provide:

1. Calibrated spectral line data cubes for each observed source.

2. Maps of each molecular species (plus channel maps in the case of gradients).

3. Identifications for each emission line seen in the full spectrum of the central point in each source.

4. Gas temperatures and column densities for the important species in each source.

After the proprietary period has ended, all data products will be available from the archive at the Canadian Astronomy Data Centre (CADC) ${ }^{26}$ More details about the SLS and its progress will be available on the survey Web site. ${ }^{27}$

\section{ANCILLARY DATA}

Despite the wealth of information contained in a spectral survey, observations of a single-frequency band have a number of limitations. Clearly, there are species that do not have any transitions in a particular frequency range. As an example, $\mathrm{DCO}^{+}$ and $\mathrm{H}_{2} \mathrm{D}^{+}$do not have any transitions in the $330-360 \mathrm{GHz}$ window covered by the SLS. There are also many species, particularly diatomic and linear species, that have only a single transition in a typical frequency band. Deriving the column density of such molecules requires more assumptions than for species with multiple transitions within the band. In addition, there may well be an excitation mismatch between the transitions of different molecules that are observed in the band. For example, the upper state energy of the CO $J=3-2$ transition, the $\mathrm{CO}$ line in the HARP-B band, is $32 \mathrm{~K}$ above ground, whereas the lowest level of any $\mathrm{CH}_{3} \mathrm{CCH}$ transition in the band is $170 \mathrm{~K}$ above ground. So if there is a range of temperatures along a line of sight, these two species are sensitive to different material. To overcome these limitations requires observations at other frequencies.

\footnotetext{
${ }^{26}$ See http://cadcwww.dao.nrc.ca.

${ }^{27}$ See http://www.jb.man.ac.uk/research/sls.
}

TABLE 2

SLS Source List and Total Integration Times

\begin{tabular}{|c|c|c|c|c|}
\hline Source & R.A. (J2000.0) & Decl. (J2000.0) & Ave. Elevation & $\begin{array}{l}\text { Total Int. Time } \\
\text { (hr) }\end{array}$ \\
\hline NGC 1333 IRAS 4 & 032911.25 & +311319.5 & $60^{\circ}$ & 115.6 \\
\hline W49 ..................... & 191013.4 & +090614 & $60^{\circ}$ & 16.8 \\
\hline AFGL $2591 \ldots \ldots \ldots \ldots$ & 202924.9 & +401120 & $60^{\circ}$ & 16.8 \\
\hline IRAS $20126+4104 \ldots \ldots$ & 201426.0 & +411332 & $60^{\circ}$ & 16.8 \\
\hline Orion Bar ................. & 053522.5 & -052500 & $50^{\circ}$ & 18.8 \\
\hline Total time: & & & & 184.8 \\
\hline
\end{tabular}

Note. - Units of right ascension are hours, minutes, and seconds, and units of declination are degrees, arcminutes, and arcseconds. 
TABLE 3

Species Detected and Imaged by the SLS Survey at $230 \mathrm{GHz}$

\begin{tabular}{lc}
\hline \hline \multicolumn{1}{c}{ Source } & \multicolumn{1}{c}{ Species } \\
\hline W49 $\ldots \ldots \ldots \ldots \ldots \ldots \ldots$ & ${ }^{13} \mathrm{CO}, \mathrm{C}^{18} \mathrm{O}, \mathrm{C}^{17} \mathrm{O}, \mathrm{HCN}, \mathrm{H}^{13} \mathrm{CN}, \mathrm{H}^{13} \mathrm{CO}^{+}, \mathrm{CH}_{3} \mathrm{OH}, \mathrm{SiO}$ \\
Orion Bar $\ldots \ldots \ldots \ldots \ldots$. & ${ }^{13} \mathrm{CO}, \mathrm{C}^{18} \mathrm{O}, \mathrm{C}^{17} \mathrm{O}, \mathrm{HCN}$ \\
NGC 1333 IRAS $4 \ldots \ldots$. & ${ }^{13} \mathrm{CO}, \mathrm{C}^{18} \mathrm{O}, \mathrm{C}^{17} \mathrm{O}, \mathrm{HCN}, \mathrm{H}^{13} \mathrm{CO}^{+}, \mathrm{CH}_{3} \mathrm{OH}, \mathrm{DCO}^{+}$, \\
AFGL $2591 \ldots \ldots \ldots \ldots \ldots$ & ${ }^{13} \mathrm{CO}, \mathrm{C}^{18} \mathrm{O}, \mathrm{HCN}, \mathrm{H}^{13} \mathrm{CN}, \mathrm{H}^{13} \mathrm{CO}^{+}, \mathrm{CH}_{3} \mathrm{OH}$ \\
IRAS 20126 $\ldots \ldots \ldots \ldots$. & ${ }^{13} \mathrm{CO}, \mathrm{C}^{18} \mathrm{O}, \mathrm{C}^{17} \mathrm{O}, \mathrm{DCN}, \mathrm{CH}_{3} \mathrm{OH}, \mathrm{H}_{2} \mathrm{~S}(?)$ \\
\hline
\end{tabular}

The SLS has already started to propose for and collect such observations to help address these issues in the analysis of the SLS data. For example, we have already obtained a set of maps covering the HARP-B field of view in a number of species at $230 \mathrm{GHz}$ (see Table 3). In addition, a full spectral scan from 211 to $279 \mathrm{GHz}$ at a single position has been obtained toward AFGL 2591. We have also been awarded time at the Green Bank Telescope (GBT) to obtain low-frequency spectral scans of the sources covering the range $8-26 \mathrm{GHz}$. This frequency range covers transitions of species, including $\mathrm{HC}_{3} \mathrm{~N}, \mathrm{HC}_{5} \mathrm{~N}$ and $\mathrm{HC}_{7} \mathrm{~N}$, $\mathrm{HDO}, \mathrm{C}_{4} \mathrm{H}, \mathrm{C}_{6} \mathrm{H}, \mathrm{CCS}, \mathrm{H}_{2} \mathrm{CO}, \mathrm{N}_{3} \mathrm{H}$, and the ground-state transitions of $\mathrm{CH}_{3} \mathrm{CCH}$ and $\mathrm{CH}_{3} \mathrm{CN}$. These GBT spectral scans will be made at a single position toward the central source. For comparison with the SLS observations, the HARP-B data will be convolved down to the GBT beam size, which over the frequency range to be observed varies from 84 " to $30^{\prime \prime}$.

In addition to these ancillary data, we have also been awarded $76 \mathrm{hr}$ of grade $1-2$ weather time (i.e., $0.5 \mathrm{~mm}<\mathrm{PWV}<1.75$ $\mathrm{mm}$ ) at the JCMT in 2007. This time will be used to observe the SLS sources at frequencies between 360 and $373 \mathrm{GHz}$, which, as Figure 1 illustrates, requires good weather conditions. The 360-373 GHz spectral region is the most poorly observed part of the $345 \mathrm{GHz}$ window, but is known to contain transitions of deuterated species $\left(\mathrm{DCO}^{+}, \mathrm{DCN}\right.$, and $\left.\mathrm{H}_{2} \mathrm{D}^{+}\right)$, formaldehyde, and methanol, together with sulfur-bearing species, which can probe different evolutionary stages of the star formation process. These data, together with any other data taken by the SLS collaboration, will also be made publicly available.

\section{REFERENCES}

André, P., Ward-Thompson, D., \& Barsony, M. 1993, ApJ, 406, 122 Bieging, J. H., Wilson, T. L., \& Downes, D. 1982, A\&AS, 49, 607

Blake, G. A., Mundy, L. G., Carlstrom, J. E., Padin, S., Scott, S. L., Scoville, N. Z., \& Woody, D. P. 1996, ApJ, 472, L49

Blake, G. A., Sandell, G., van Dishoeck, E. F., Groesbeck, T. D., Mundy, L. G., \& Aspin, C. 1995, ApJ, 441, 689

Blake, G. A., Sutton, E. C., Masson, C. R., \& Phillips, T. G. 1987, ApJ, 315, 621

Bottinelli, S., et al. 2004, ApJ, 615, 354

Buckle, J. V., \& Fuller, G. A. 2003, A\&A, 399, 567

Buckle, J. V., et al. 2007, in IAU Symp. 237, Triggered Star Formation in a Turbulent ISM, in press

Caselli, P., van der Tak, F. F. S., Ceccarelli, C., \& Bacmann, A. 2003, A\&A, 403, L37

Cazaux, S., et al. 2003, ApJ, 593, L51

Ceccarelli, C., et al. 2000, A\&A, 355, 1129

Cesaroni, R., Neri, R., Olmi, L., Testi, L., Walmsley, C. M., \& Hofner, P. 2005, A\&A, 434, 1039

Charnley, S. B. 1997, ApJ, 481, 396

Choi, M. 2001, ApJ, 553, 219 2005, ApJ, 630, 976

Cohen, N. L., \& Willson, R. F. 1981, A\&A, 96, 230

Comito, C., Schilke, P., Phillips, T. G., Lis, D. C., Motte, F., \& Mehringer, D. 2005, ApJS, 156, 127

de Zeeuw, P. T., Hoogerwerf, R., de Bruijne, J. H. J., Brown, A. G. A., \& Blaauw, A. 1999, AJ, 117, 354

Di Francesco, J., Myers, P. C., Wilner, D. J., Ohashi, N., \& Mardones, D. 2001, ApJ, 562, 770

Doty, S. D., Schöier, F. L., \& van Dishoeck, E. F. 2004, A\&A, 418, 1021

Doty, S. D., van Dishoeck, E. F., van der Tak, F. F. S., \& Boonman, A. M. S. 2002, A\&A, 389, 446
Estalella, R., Mauersberger, R., Torrelles, J. M., Anglada, G., Gomez, J. F., Lopez, R., \& Muders, D. 1993, ApJ, 419, 698

García-Burillo, S., et al. 2002, ApJ, 575, L55

Genzel, R., et al. 1978, A\&A, 66, 13

Goicoechea, J. R., Rodríguez-Fernández, N. J., \& Cernicharo, J. 2004, ApJ, 600, 214

Greaves, J. S., \& Williams, P. G. 1994, A\&A, 290, 259

Groesbeck, T. D. 1995, Ph.D. thesis, Caltech

Gwinn, C. R., Moran, J. M., \& Reid, M. J. 1992, ApJ, 393, 149

Harvey, P. M., Campbell, M. F., \& Hoffmann, W. F. 1977, ApJ, 211, 786

Hatchell, J., Roberts, H., \& Millar, T. J. 1999, A\&A, 346, 227

Hatchell, J., Thompson, M. A., Millar, T. J., \& Macdonald, G. H. 1998a, A\&AS, 133, 29 1998b, A\&A, 338, 713

Helmich, F. P., \& van Dishoeck, E. F. 1997, A\&AS, 124, 205

Hogerheijde, M. R., Jansen, D. J., \& van Dishoeck, E. F. 1995, A\&A, 294, 792

Hogerheijde, M. R., \& van der Tak, F. F. S. 2000, A\&A, 362, 697

Holland, S. E., et. al. 2006, Proc. SPIE, 6275, 45

Homeier, N. L., \& Alves, J. 2005, A\&A, 430, 481

Jansen, D. J., Spaans, M., Hogerheijde, M. R., \& van Dishoeck, E. F. 1995, A\&A, 303, 541

Jørgensen, J. K., Schöier, F. L., \& van Dishoeck, E. F. 2004, A\&A, 416, 603

. 2005, A\&A, 437, 501

Lay, O. P., Carlstrom, J. E., \& Hills, R. E. 1995, ApJ, 452, L73

Lebrón, M., Beuther, H., Schilke, P., \& Stanke, T. 2006, A\&A, 448, 1037

Leurini, S., et al. 2006, A\&A, 454, L47

Looney, L. W., Mundy, L. G., \& Welch, W. J. 2000, ApJ, 529, 477

Lovas, F. 2004, J. Phys. Chem. Ref. Data, 33, 177

Lucas, R., \& Liszt, H. S. 2000, A\&A, 355, 327 
Macdonald, G. H., Gibb, A. G., Habing, R. J., \& Millar, T. J. 1996, A\&AS, 119, 333

Maret, S., et al. 2004, A\&A, 416, 577

Martín, S., et al. 2005, ApJ, 620, 210

McKee, C. F., \& Tan, J. C. 2002, Nature, 416, 59

Mitchell, G. F., Hasegawa, T. I., \& Schella, J. 1992, ApJ, 386, 604

Mitchell, G. F., Maillard, J.-P., Allen, M., Beer, R., \& Belcourt, K. 1990, ApJ, 363, 554

Mufson, S. L., \& Liszt, H. S. 1977, ApJ, 212, 664

Nomura, H., \& Millar, T. J. 2004, A\&A, 414, 409

Nummelin, A., Bergman, P., Hjalmarson, A., Friberg, P., Irvine, W. M., Millar, T. J., Ohishi, M., \& Saito, S. 1998, ApJS, 117, 427

Nyman, L. A. 1983, A\&A, 120, 307

Osamura, Y., Roberts, H., \& Herbst, E. 2004, A\&A, 421, 1101

Pardo, J. R., et al. 2001, ApJ, 562, 799

Parise, B., et al. 2005, A\&A, 431, 547

Plume, R., et al. 2004, ApJ, 605, 247

Radhakrishnan, V., Goss, W. M., Murray, J. D., \& Brooks, J. W. 1972, ApJS, 24, 49

Rodgers, H., \& Millar, T. 1996, MNRAS, 280, 1046

Savage, C., \& Ziurys, L. M. 2004, ApJ, 616, 966

Schilke, P., Groesbeck, T. D., Blake, G. A., \& Phillips, T. G. 1997, ApJS, 108, 301

Schilke, P., Pineau des Forets, G., Walmsley, C. M., \& Martin-Pintado, J. 2001, A\&A, 372, 291

Schneider, N., Bontemps, S., Simon, R., Jakob, H., Motte, F., Miller, M., Kramer, C., \& Stutzki, J. 2006, A\&A, 458, 855

Schöier, F. L., Jørgensen, J. K., van Dishoeck, E. F., \& Blake, G. A. 2004, A\&A, 418, 185

Schöier, F. L., van der Tak, F. F. S., van Dishoeck, E. F., \& Black, J. H. 2005, A\&A, 432, 369
Shepherd, D. S., Yu, K. C., Bally, J., \& Testi, L. 2000, ApJ, 535, 833

Shu, F. H., Adams, F. C., \& Lizano, S. 1987, ARA\&A, 25, 23

Simon, R., Jackson, J. M., Clemens, D. P., Bania, T. M., \& Heyer, M. H. 2001, ApJ, 551, 747

Smith, H., et al. 2003, Proc. SPIE, 4855, 338

Sridharan, T. K., Williams, S. J., \& Fuller, G. A. 2005, ApJ, 631, L73

Stark, R., van der Tak, F. F. S., \& van Dishoeck, E. F. 1999, ApJ, 521, L67

Stäuber, P., Benz, A. O., Jørgensen, J. K., van Dishoeck, E. F., Doty, S. D., \& van der Tak, F. F. S. 2007, A\&A, in press (astro-ph/ 0608393)

Sutton, E. C., et al. 1991, ApJS, 77, 255

Thompson, M. A., \& Macdonald, G. H. 1999, A\&AS, 135, 531

Trinidad, M. A., et al. 2003, ApJ, 589, 386

van der Tak, F. F. S., Boonman, A. M. S., Braakman, R., \& van Dishoeck, E. F. 2003, A\&A, 412, 133

van der Tak, F. F. S., van Dishoeck, E. F., \& Caselli, P. 2000, A\&A, 361,327

van der Tak, F. F. S., van Dishoeck, E. F., Evans, N. J., II, Bakker, E. J., \& Blake, G. A. 1999, ApJ, 522, 991

van der Tak, F. F. S., Walmsley, C. M., Herpin, F., \& Ceccarelli, C. 2006, A\&A, 447, 1011

van Dishoeck, E. F., \& Blake, G. A. 1998, ARA\&A, 36, 317

van Dishoeck, E. F., Blake, G. A., Jansen, D. J., \& Groesbeck, T. D. 1995, ApJ, 447, 760

Wakelam, V., et al. 2004, A\&A, 422, 159

Ward-Thompson, D., \& Robson, E. 1990, MNRAS, 244, 458

White, G. J., Araki, M., Greaves, J. S., Ohishi, M., \& Higginbottom, N. S. 2003, A\&A, 407, 589 